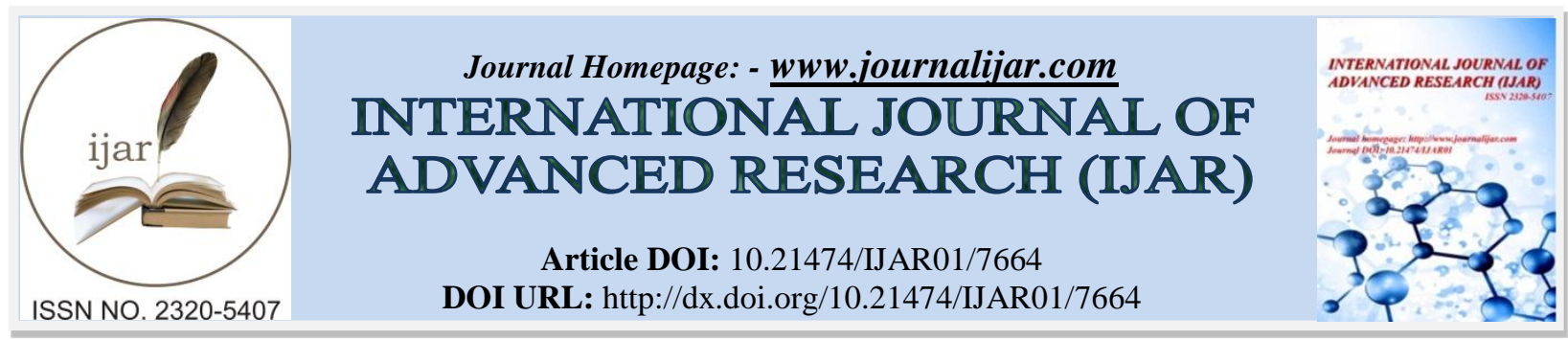

RESEARCH ARTICLE

\title{
LEUKOARAIOSIS AND DEPRESSION: LINKS BETWEEN THE RADIOLOGICAL ENTITY AND THE DEPRESSIVE SYMPTOMATOLOGY.
}

\section{Anouar Kaddaf, Yassine Otheman, Youness Mamouni Alaoui, Mohamed Kadiri, Jamal Mehssani and Mohamed zakarya Bichra. \\ Department of Psychiatry, Military Hospital of Instruction Mohammed V, Rabat, Morocco.}

\section{Manuscript Info}

Manuscript History

Received: 04 July 2018

Final Accepted: 06 August 2018

Published: September 2018

Keywords:-

Leukoaraiosis, vascular depression,

depressive symptomatology

\section{Abstract}

Leukoaraiosis is a purely descriptive term for a radiological image, which describe confluent white matter abnormalities, detected by computed tomography (CT) or magnetic resonance imaging (MRI). The clinical presentation of this entity is heterogeneous and mostly severe. The relationship between Leukoaraiosis and depressive symptomatology is not yet completely understood. Ischemia seems to be the main favoring factor. Leukoaraiosis may be a risk factor for the onset, persistence, or worsening of depressive symptomatology in the elderly. Through a clinical case, we will address the different clinical and therapeutic aspects of this relationship.

Copy Right, IJAR, 2018,. All rights reserved.

\section{Introduction:-}

Leukoaraiosis is a purely descriptive term for a radiological image, which appeared in the literature in the mid-1980s to describe confluent white matter abnormalities, detected by computed tomography (CT) or magnetic resonance imaging (MRI). Today, we know that the clinical presentation of this entity is heterogeneous and mostly severe; it can take the form of vascular dementia, or present as urinary disorders, cognitive or mood disturbances. It is also associated with a higher risk of ischemic stroke and cerebral hemorrhage. The relationship between Leukoaraiosis and depressive symptomatology is not yet completely understood. Through a clinical case, we will address the different clinical and therapeutic aspects of this relationship.

\section{Clinical case:-}

$\mathrm{Mr}$ A.l. is a 78 years oldman, without psychiatric history. He reported 4 years ago the occurrence of frontal headaches, such as electric shock, resistant to the usual analgesics, accompanied by buzzing and rotary vertigo when changing position. His family also reported memory disturbances. The general examination had found normal blood pressure, while MMSE (Mini Mental State Evaluation) was at 27/30. Biological assessment revealed a fasting blood sugar (FBS) at $1.30 \mathrm{~g} / \mathrm{l}$. Electrocardiogram (ECG), transthoracic echocardiography (TTE) and blood pressure holter were normal; whereas an echocardiogram of the neck vessels had objectified small atheromatous plaques located at the level of the two carotid bulbs, with stenosis at the right external carotid artery. CT showed a moderate periventricular leukoaraiosis, confirmed by brain MRI (figures 1,2)

The patient received Gliclazide $30 \mathrm{mg} /$ day, simvastatin $20 \mathrm{mg} /$ day, acetylleucine $1.5 \mathrm{~g} /$ day, carvedilol $6.25 \mathrm{mg} /$ day and aspirin100mg/day, with a good evolution. 
A year ago, the patient refused his medications, in a context of sadness of the mood with insomnia, pessimistic thoughts and loss of appetite. The diagnosis of a characterized depression episode was made, and the patient received antidepressant (fluoxetine: $20 \mathrm{mg} /$ day) and anxiolytic (lorazepam: $1 \mathrm{mg} / \mathrm{night}$ for two weeks). The patient has evolved well after one month of treatment, and he resumed his follow-up and his treatments.

\section{Discussion:-}

Late onset depression is known by its association with vascular risk factors, cognitive decline, cerebral atrophy and leukoaraiosis lesions in MRI [1,2].

Studies suggest a relationship between depressive symptomatology and the localization and the severity of Leukoaraiosis [3]. This relationship has been better described when it comes to the hyper signals observed in gray nuclei compared to those observed in white matter [4,5].

The vascular origin of depression in patients with leukoaraiosis is frequently reported, given the similarity of risk factors predisposing or precipitating leukoaraiosis to those of atherosclerotic vascular disease [1]. This was at the origin of the notion of vascular depression, which associates depressive episode to leukoaraiosis lesions on MRI according to the Fazekas classification [6].

Several hypotheses have been advanced to explain these links. First, the reduction of the plasticity of the vessels irrigating white matter is responsible of a hypo-infusion, insufficient to create an infarction but capable of damaging the parenchyma [7-9], which could lead to a frontal subcortical dysfunction [1]. These anomalies associated with striato-frontal aging (leading to cognitive disorders) and psychosocial factors are responsible for a depressive vulnerability [10].

Another hypothesis insists on the role of the extravasation of serum proteins in the interstitial space (by endothelial dysfunction), such as the adhesive molecule intercellular-1 and vascular adhesive-1, found in the dorso-lateral prefrontal cortex of the depressed subjects in greater quantity. The links between these markers of ischemia and depressive state is probably due to interactions with the hypothalamic axis and inflammatory factors such as cytokines [1].

In the case we reporting here, the absence of arterial hypertension (the main risk factor incriminated in the hypothesis of vascular depression), can be explained by the fact that the patient had carotid atherosclerosis, which could have the same consequences as hypertension on the cerebral blood flow. Alternatively, the hypothesis of the markers of ischemia may also be an explanation, knowing that the presence of these markers would be independent of the existence or not of an arterial hypertension [11].

\section{Conclusion:-}

Leukoaraiosis may be a risk factor for the onset, persistence, or worsening of depressive symptomatology in the elderly [3]. The relationship between Leukoaraiosis and depression is still not fully understood. Ischemia seems to be the main favoring factor, but other hypotheses incriminating the hypothalamic axis and cytokines are also serious leads.

\section{Figures}

Figure 1:-Cerebral MRI FLAIR axial section of the patient, showing periventricular white matter signal abnormalities in moderate periventricular leukoaraiosis.

Figure 2:-Cerebral MRI T2 coronal section of the patient, showing periventricular white matter signal abnormalities in moderate periventricular leukoaraiosis

\section{Conflicts of interest:-}

The authors do not declare any conflict of interest for this work.

Authors' collaboration:-

All authors were actively involved in the development of this work. 


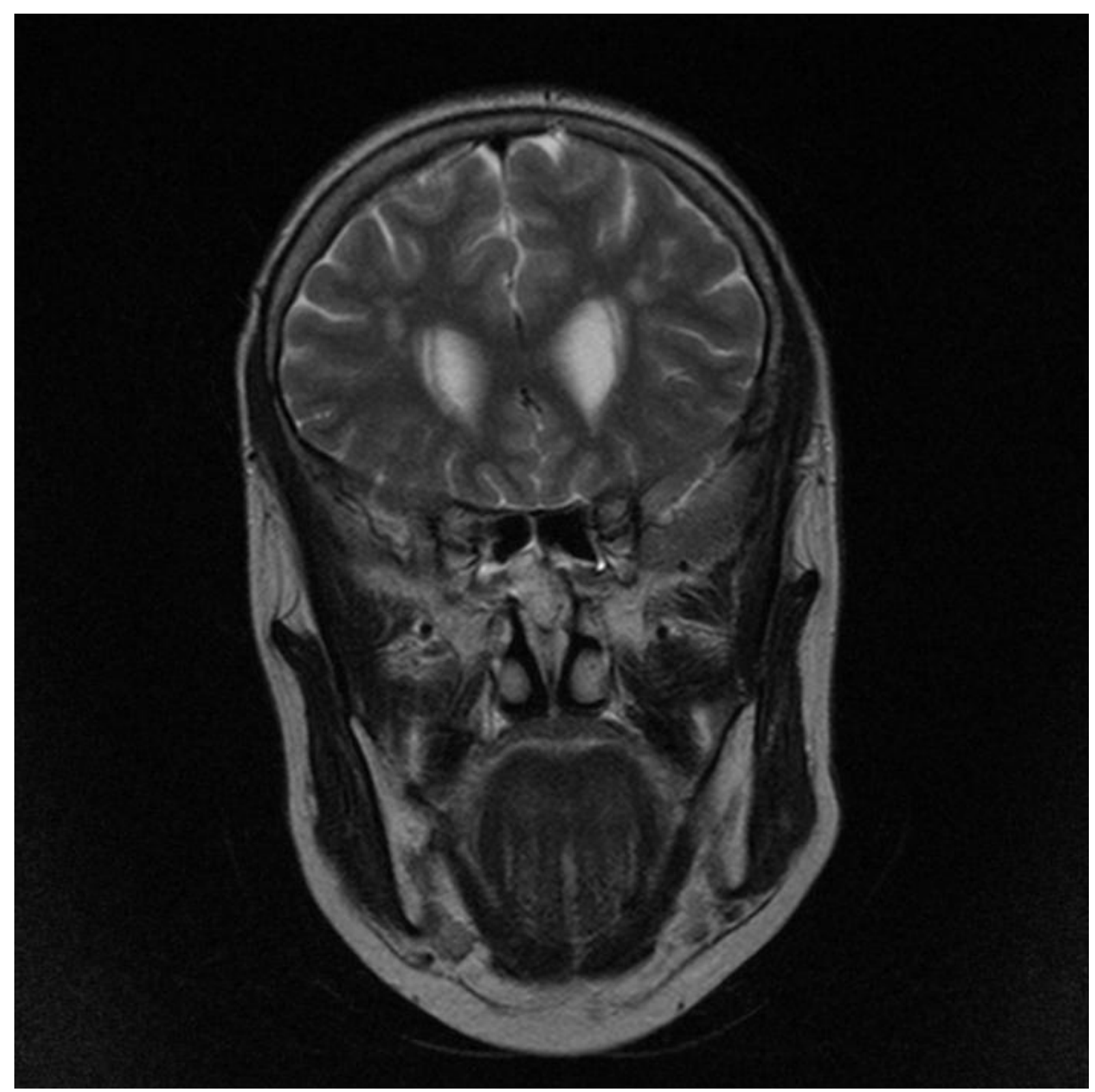

Figure 1:-Cerebral MRI FLAIR axial section of the patient, showing periventricular white matter signal abnormalities in moderate periventricular leukoaraiosis. 


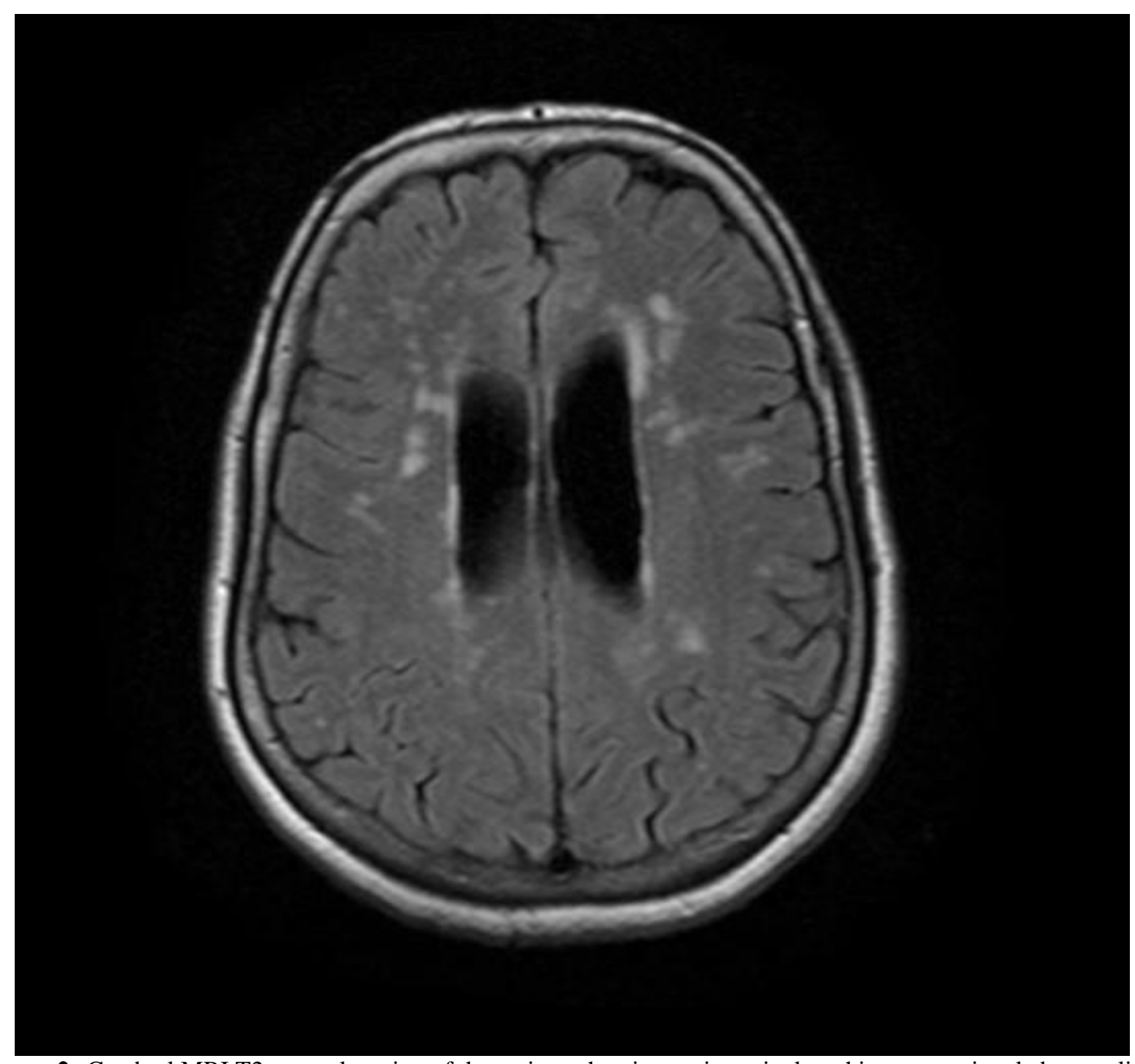

Figure 2:-Cerebral MRI T2 coronal section of the patient, showing periventricular white matter signal abnormalities in moderate periventricular leukoaraiosis. 


\section{References:-}

1. Lebert F. Dépression vasculaire, limites du concept. Psychol NeuroPsychiatr Vieillissement $2004 ; 3: 173-9$.

2. Baldwin RC, O’Brien J. Vascular basis of late onset depressive episode. Br J Psychiatry 2002 ; 180 : 157-60.

3. Steffens DC, Krishnan KR, Crump C, Burke GL. Cerebrovascular disease and evolution of depressive symptoms in the cardiovascular health study. Stroke $2002 ; 33: 1636-44$.

4. Steffens DC, Helm MJ, Krishnan KR, Burke GL. Cerebrovascular disease and depression symptoms in the cardiovascular health study. Stroke $1999 ; 30: 2159-66$.

5. Thomas AJ, O'Brien JT, Davis S, Ballard C, Barber R, Kalaria RN. Ischemic basis for deep white matter hyperintensities in major depression: a neuropathological study. Arch Gen Psychiatry $2002 ; 59$ : 785-92.

6. Krishnan KR, Taylor WD, McQuoid DR, MacFall JR, Paynette ME, Provenzale JM, et al. Clinical characteristics of magnetic resonance imaging-defined subcortical ischemic depression. Biol Psychiatry 2004 ; $55: 390-7$.

7. Nadeau Y, Verreault S. La leucoaraïose : plus qu'une découverte fortuite. Le clinicien 2010; 51-54.

8. Kurumatani T, Kudo T, Ikura Y, Takeda M. White matter changes in the gerbil brain under chronic cerebral hypoperfusion. Stroke $1998 ; 29: 1058-62$.

9. Cottier J.-P et al. Pathology of small cerebral arteries demonstrated by MRI: a marker of aging? Geriatr Psychol Neuropsychiatr Vieil $2011 ; 9: 465-75$.

10. Pugh KG, Lipsitz LA. The microvascular frontal-subcortical syndrome of aging. Neurobiol Aging $2002 ; 23$ : 421-31.

11. Thomas AJ, Perry R, Kalaria R, Oakley A, McMeekin W, O'Brien J. Neuropathological evidence for ischemia in the whitte matter of the dorsolateral prefrontal cortex in late-life depression. Int J Geriatr Psychiatry 2003 ; $18: 7-13$. 